\title{
Periodontal disease and glycemic control in diabetics
}

\author{
Abstracted from
}

Corbella S, Francetti L, Taschieri S, De Siena F, Fabbro MD.

Effect of periodontal treatment on glycemic control of patients with diabetes: A systematic review and meta-analysis. J Diabetes Investig 2013; 4: 502-509.

Address for correspondence: Stefano Corbella, Oral Implantology Research Center,

Department of Biomedical, Surgical and Dental Sciences, Universit_a degli Studi di Milano, IRCCS Istituto Ortopedico Galeazzi, Milan, Italy. E-mail: stefano.corbella@gmail.com

\section{Question: Does non-surgical periodontal treatment improve glycaemic control in diabetic patients?}

Data sources Medline, Embase and the Cochrane Central Register of Controlled Trials (CENTRAL). This was supplemented by handsearching of the Journal of Clinical Periodontology, Journal of Periodontology, Journal of Dental Research, Journal of Dentistry, Journal of Periodontal Research, International Journal of Periodontics and Restorative Dentistry, Periodontology 2000, Odontology, Clinical Oral Investigations, Annals of Periodontology, Journal of American Dental Association, British Dental Journal, Community Dentistry and Oral Epidemiology, Diabetes, Diabetes Care, Diabetes \& Metabolic Syndrome, Diabetes \& Metabolism and Annals of Internal Medicine. There were no language restrictions.

Study selection Randomised controlled trials (RCTs) on diabetic patients with periodontal disease that reported glycated haemoglobin (HbA1c) and/or fasting plasma glucose (FPG) modification after treatment, with a minimum of three months follow-up were included. Study quality was assessed independently by two reviewers.

Data extraction and synthesis Data extraction was carried out independently by two reviewers. Meta-analysis was carried out to evaluate the effect of non-surgical periodontal treatment on $\mathrm{HbA} 1 \mathrm{c}$ and FPG levels. The effect of the adjunctive use of antimicrobials was also assessed

Results Fifteen studies were included, five were considered to be at low risk of bias, three at high risk and the remainder at unclear risk. Seven studies (678 patients) contributed to the meta-analysis for HbA1c. At the three-four months follow-up, the weighted mean difference was $-0.38 \%(95 \% \mathrm{Cl}-0.23$ to $-0.53 ; \mathrm{P}<0.001)$ and at six months it was $-0.31 \%(95 \% \mathrm{Cl} 0.11$ to $-0.74 ; \mathrm{P}=0.15)$, favouring the treatment groups [three studies contributing to meta-analysis]. There was statistically significant heterogeneity for both comparisons. For FPG, five studies presented data for three-four months and two for six months. At the three-four months follow-up, the weighted mean difference was $-9.01 \mathrm{mg} / \mathrm{dL}(95 \% \mathrm{Cl}-2.24$ to $-15.78 ; \mathrm{P}=0.009)$ and at six months it was $-13.62 \mathrm{mg} / \mathrm{dL}(95 \% \mathrm{Cl} 0.45$ to $-27.69 ; \mathrm{P}=0.06)$.

Conclusions Despite the limitations of the present study, it can be concluded that periodontal treatment might be effective in improving metabolic control in terms of reduction of $\mathrm{HbA} 1 \mathrm{c}$ and FPG concentrations in patients with diabetes. However, the significance of this improvement is questionable and should be further investigated.

\section{Commentary}

Corbella and colleagues have prepared a systematic review and meta-analysis of clinical trials that addresses whether periodontal therapy influences glycaemic control. The authors adhered to standard methods as described by the Cochrane group, and used Rev Man (5.1) for data analysis. Manuscripts were selected according to predetermined criteria, and included studies in which a randomisation protocol was followed that included a non-surgical periodontal intervention and a diabetes outcome, either haemoglobin A1c (HbA1c) or Fasting Plasma Glucose (FPG), and a follow up of at least three months. While PRISMA guidelines ${ }^{1}$ were not referenced in the text, a transparent data extraction and analysis methodology were described.

Meta-analyses were conducted on HbA1c or FPG outcomes at three or six months, and studies were analysed separately according to risk of bias evaluation. Heterogeneity between studies was calculated in order to determine whether publication bias was significant. ${ }^{2}$ In the main analysis that included 678 subjects from eight studies, a small but statistically significant reduction in HbA1c was seen favouring periodontal treatment after three months. Studies conducted over six months did not show a significant reduction in HbA1c. Likewise, studies that looked at FPG showed a significant reduction at three months, but not at six months. Studies that included adjunctive antimicrobials, when taken together did not result in significant differences in HbA1c. In both groups of studies, significant heterogeneity was noted.

This analysis was well conducted, although the manuscript copy I received had mismatched Figure legends (presumably this will be corrected by the authors in due course). Nevertheless this metaanalysis has important limitations and the results should be interpreted with caution. While the number of studies that address the role of periodontal therapy and diabetes outcomes is growing of late, the individual studies themselves are underpowered to address the central study question of whether non-surgical periodontal therapy reduces short-term measures of HbA1c. Simply adding up the small studies and averaging the outcome, as is done in metaanalysis, is not the same as conducting a large randomised trial of similar sample size. ${ }^{3}$ For a meta-analysis to be high quality evidence, each of the trials included must also be of high quality, and that means each must have sufficient statistical power on its own. Indeed the recently completed Diabetes and Periodontal Therapy Trial, which had $90 \%$ power to answer the study question, failed to demonstrate a benefit to diabetes outcomes following non-surgical periodontal therapy. ${ }^{4}$ Time will tell whether other periodontal 


\section{PERIODONTAL DISEASE}

treatment protocols may improve diabetes outcomes, but until other large randomised clinical trials show otherwise, the best available evidence does not suggest that short-term diabetes outcomes are improved following non-surgical periodontal therapy.

\section{Steven Engebretson}

Department of Periodontology and Implant Dentistry, New York University, New York, USA

1. Liberati A, Altman DG, Tetzlaff J, et al. The PRISMA statement for reporting systematic reviews and meta-analyses of studies that evaluate health care interventions: explanation and elaboration. PLoS Med 2009; 6: e1000100.

2. Higgins JP, Thompson SG. Quantifying heterogeneity in a metaanalysis. Stat Med 2002; 21: 1539-1558.

\section{Practice points}

- The results of a large randomised control trial did not confirm the findings of systematic reviews made up of underpowered studies.

- Periodontal therapy is beneficial to patients with diabetes, but not as a means of improving glycaemic control.

3. LeLorier J, Gregoire G, Benhaddad A, Lapierre J, Derderian F. Discrepancies between meta-analyses and subsequent large randomized, controlled trials. N Engl / Med 1997; 337: 536-542.

4. Engebretson SP, Hyman LG, Michalowicz BS, Schoenfeld ER, Gelato MC, Hou W, et al. The effect of nonsurgical periodontal therapy on hemoglobin A1c levels in persons with type 2 diabetes and chronic periodontitis: a randomized clinical trial. / Am Med Assoc 2013; 310: 2523-2532.

Evidence-Based Dentistry (2014) 15, 93-94. doi:10.1038/sj.ebd.6401040 